\title{
Rendement en viande de poulets de deux lignées sélectionnées sur l'état d'engraissement
}

\author{
F.-H. RICARD, B. LECLERCQ et G. MARCHÉ \\ I.N.R.A., Station de Recherches Avicoles, Centre de Recherches de Tours, Nouzilly, \\ F 37380 Monnaie
}

\begin{abstract}
Résumé
Les caractéristiques de carcasse de poulets appartenant à deux lignées expérimentales, soumises à une sélection divergente sur l'importance du dépôt gras abdominal, ont été étudiées sur trois échantillons représentant au total 52 animaux pour la lignée "grasse " et 63 pour la lignée «maigre ». Dans la lignée maigre, le dépôt gras abdominal, exprimé en pour-cent du poids vif, est diminué de 1,4 à 2,4 points selon les échantillons. En contrepartie, le pourcentage de la carcasse éviscérée est augmenté de 0,6 à 2,2 points. Une dissection des principaux éléments de la carcasse a été faite pour deux des échantillons étudiés : dans la lignée maigre, le poids des muscles nobles (poitrine, cuisses et pilons) rapporté au poids de la carcasse éviscérée est augmenté de 2,1 à 2,9 p. 100 . L'ensemble des résultats obtenus montre qu'une sélection pour diminuer les dépôts gras abdominaux s'accompagne d'une amélioration significative du rendement en viande chez le poulet.
\end{abstract}

L'état d'engraissement du poulet peut se mesurer de façon simple par l'importance relative des dépôts gras abdominaux (DELPECH \& RiCARD, 1965). L'héritabilité de ce caractère est élevée (RICARD \& Rouvier, 1967) et une expérience de sélection divergente a permis d'obtenir dans notre station deux lignées de poulets qui diffèrent fortement pour l'état d'engraissement (LECLERCQ et al., 1980). On peut se demander quelles sont les conséquences d'une telle sélection sur les autres caractéristiques de carcasse. Nous présentons ici quelques résultats concernant le rendement à l'abattage et le rendement en viande.

Les poulets étudiés appartiennent aux lignées "grasse » et " maigre " sélectionnées par LECLERCQ et al. (1980) à partir d'une population synthétique de type chair (BOYER, communication personnelle). Les animaux des deux lignées ont été élevés ensemble et ont donc subi les mêmes conditions d'environnement. Ils proviennent de 3 expériences indépendantes.

Expérience 1 : coquelets et poulettes abattus en novembre 1979 à l'âge de 9 semaines. Ils ont été pesés immédiatement avant l'abattage, après un jeûne complet de 16 heures. Après environ 4 heures de ressuage à la température de la salle d'abattage, on a disséqué et pesé les dépôts gras abdominaux (tissus adipeux situés contre la paroi de l'abdomen et autour du gésier et du ventricule succenturié), le cou (la moitié de la peau du cou est laissée avec la carcasse), les abats consommables 
(cœur + foie + gésier). Tous les autres abats et organes internes ont été enlevés pour obtenir le poids de la carcasse éviscérée.

Expérience 2 : coquelets abattus en octobre 1980 à l'âge de 9 semaines, selon le même protocole expérimental. De plus, on a prélevé les muscles pectoraux (sans la peau ni l'os du bréchet) et l'ensemble cuisses + pilons. Sur ces derniers, une dissection plus complète a été opérée pour obtenir le poids des différents tissus : peau, os, gras dissécable (dépôts sous-cutanés et entre muscles), muscles.

Expérience 3: coquelets abattus en mai 1981 à l'âge de 7 semaines et demi (52 jours), selon le même protocole d'abattage et de dissection que dans l'expérience 2 .

Les tableaux 1,2 et 3 regroupent les résultats obtenus respectivement aux cours des expériences 1,2 et 3 : moyennes, écart-types, comparaison des moyennes par le test $\mathrm{t}$ de Student. Les variances des groupes maigre et gras sont homogènes pour toutes les variables étudiées à l'exception du poids du gras abdominal dans l'expérience 3. La raison en est que la distribution de cette variable se rapproche plus d'une loi de poisson que d'une loi normale (cf. discussion de ce problème dans RICARD \& Rouvier, 1967). Pour cette raison, le test de Student a été pratiqué sur la racine carrée du poids du gras abdominal et non sur la valeur brute.

\section{TABLEAU 1}

Résultats de la première expérience, poulets âgés de 9 semaines.

Résults of the first experiment, 9-wk old broilers.

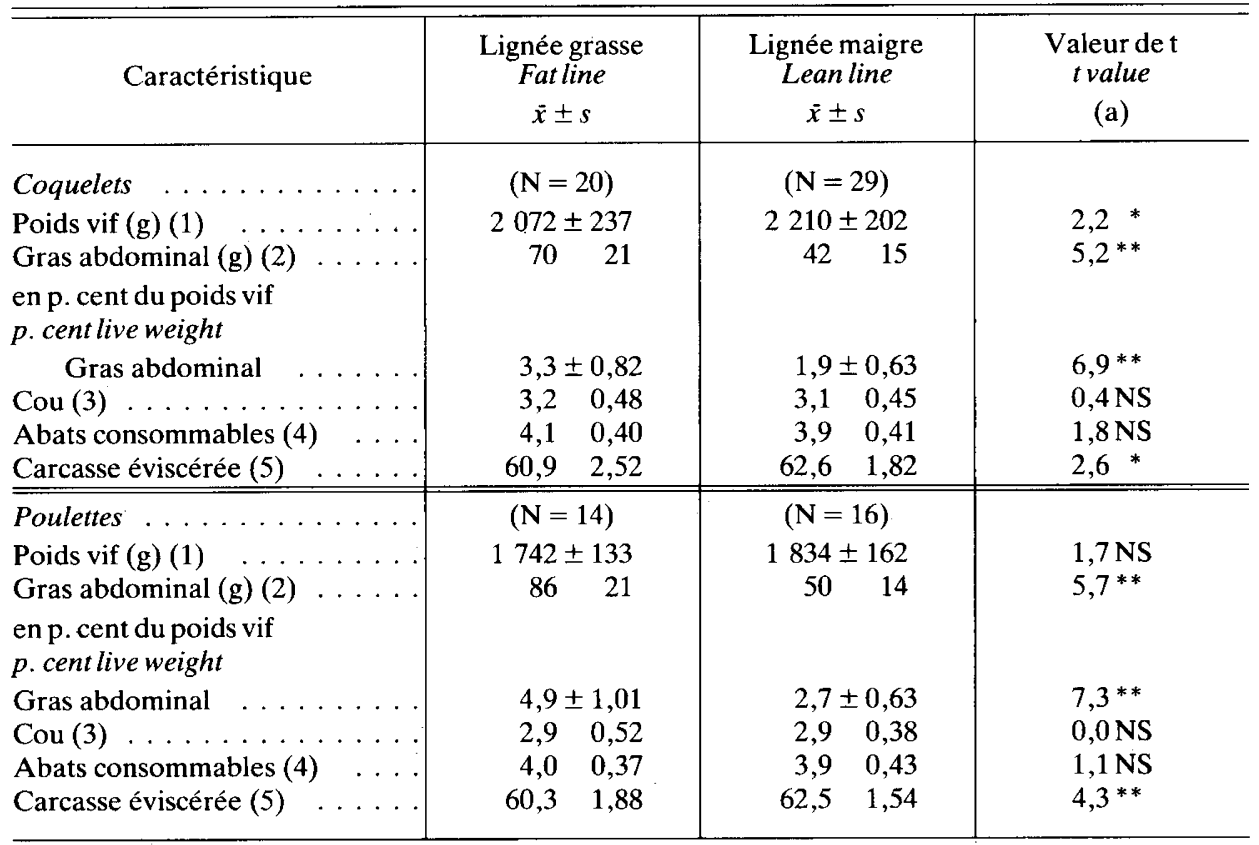

(a) NS = Différence non significative $(\mathrm{P}>0,05),{ }^{*}=\mathrm{P} \leqslant 0,05,{ }^{* *}=\mathrm{P} \leqslant 0,01$.

(1) = Live weight before slaughter, (2) = Abdominal fat, (3) = neck. (4) = Edible offals (heart + liver + gizzard), (5) $=$ Eviscerated carcass. 


\section{TABleau 2}

Résultats de la deuxième expérience, coquelets âgés de 9 semaines.

Résults of the second experiment, 9-wk old male broilers.

\begin{tabular}{|c|c|c|c|}
\hline Caractéristique & $\begin{array}{c}\text { Lignée grasse } \\
\text { Fat line } \\
\bar{x} \pm s\end{array}$ & $\begin{array}{l}\text { Lignée maigre } \\
\text { Lean line } \\
\bar{x} \pm s\end{array}$ & $\begin{array}{l}\text { Valeur de } \mathrm{t} \\
\text { tvalue }\end{array}$ \\
\hline & $(N=10)$ & $(N=10)$ & \\
\hline Poids vif $(\mathrm{g})(1)$ & $2124 \pm 76$ & $2188 \pm 209$ & $0,9 \mathrm{NS}$ \\
\hline Gras abdominal (g) (2) & $83 \quad 21$ & $35 \quad 19$ & $5,5^{* *}$ \\
\hline \multicolumn{4}{|l|}{$\begin{array}{l}\text { en } \mathrm{p} \text {. cent du poids vif } \\
\text { p. cent live weight }\end{array}$} \\
\hline Gras abdominal & $3,9 \pm 1,08$ & $1,5 \pm 0,73$ & $5,9^{* *}$ \\
\hline Cou $(3) \ldots \ldots$ & $3,4 \quad 0,12$ & $3,4 \quad 0,25$ & $0,5 \mathrm{NS}$ \\
\hline Abats consommables (4) & $3,6 \quad 0,19$ & $3,8 \quad 0,34$ & 1,4 NS \\
\hline Carcasse éviscérée (5). & $60,7 \quad 1,24$ & $61,3 \quad 1,20$ & $1,1 \mathrm{NS}$ \\
\hline \multicolumn{4}{|l|}{$\begin{array}{l}\text { en p. cent carcasse éviscérée } \\
P . \text { cent eviscerated weight }\end{array}$} \\
\hline Muscles pectoraux (6) & $18,7 \pm 0,76$ & $19,2 \pm 1,00$ & $1,4 \mathrm{NS}$ \\
\hline Muscles cuis. + pilon (7) & $24,0 \quad 1,06$ & $25,6 \quad 1,57$ & $2,6 *$ \\
\hline \multicolumn{3}{|l|}{ Muscles pectoraux } & $2,8 *$ \\
\hline \multicolumn{4}{|l|}{$\begin{array}{l}\text { en p. cent ens. cuisses }+ \text { pilons } \\
\text { p. cent thighs }+ \text { drumsticks weight }\end{array}$} \\
\hline Peau (9) $\ldots \ldots$ & $9,2 \pm 0,95$ & $9,0 \pm 0,90$ & $0,5 \mathrm{NS}$ \\
\hline Os $(10)$ & $15,5 \quad 1,12$ & $16,2 \quad 0,87$ & $1,7 \mathrm{NS}$ \\
\hline Gras dissécable (11) & $7,4 \quad 1,42$ & $4,6 \quad 1,29$ & $4,6^{* *}$ \\
\hline Muscles $\ldots \ldots \ldots$ & $61,8 \quad 1,89$ & $64,3 \quad 2,05$ & $2,08 *$ \\
\hline Tendons + pertes de dissection & $6,1-$ & $5,9-$ & - \\
\hline
\end{tabular}

(1) = Live weight before slaughter, (2) = Abdominal fat, (3) = Neck, (4) = Edible offals, (5) = Eviscerated carcass, $(6)=$ Pectoral muscles, $(7)=$ Thighs + drumsticks muscles, $(8)=$ Pectoral + Thighs + Drumsticks muscles, (9) = Skin, (10) = Bone, (11) = Dissectable fat depots.

Dans tous les échantillons, les poulets de la lignée maigre sont plus lourds que ceux de la lignée grasse, la différence étant significative pour les coquelets de l'expérience 1 . Il ne s'agit pas d'un effet d'échantillonnage mais d'une caractéristique propre aux deux lignées apparue à partir de la deuxième génération de sélection (SimoN \& LeCLeRCQ, sous presse).

Les résultats concernant le gras abdominal montrent que la sélection a été efficace : on obtient autour de 2 p. 100 de moins de gras abdominal, par rapport au poids vif, chez les poulets de la lignée maigre $(1,4$ à 2,4 p. 100 d'écart selon les échantillons). Il est intéressant de voir que cette diminution correspond pour une grande part à une augmentation du pourcentage de la carcasse éviscérée $(0,6$ à 2,2 p. 100 d'écart selon les échantillons). On peut donc dire qu'une sélection pour diminuer l'importance des dépôts gras abdominaux permet d'améliorer significativement le rendement à l'abattage, dans des proportions très intéressantes pour le bilan financier des abattoirs. La part du cou et des abats consommables a tendance à être plus faible dans la lignée maigre, mais les différences ne sont pas significatives. 


\section{TABLEAU 3}

Résultats de la troisième expérience, coquelets âgés de 7 semaines et demi.

Résults of the third experiment, $71 / 2$ wk-old male broilers.

\begin{tabular}{|c|c|c|c|}
\hline Caractéristique & $\begin{array}{l}\text { Lignée grasse } \\
\text { Fat line } \\
\bar{x} \pm s\end{array}$ & $\begin{array}{l}\text { Lignée maigre } \\
\text { Lean line } \\
\qquad \bar{x} \pm s\end{array}$ & $\begin{array}{l}\text { Valeur de } t \\
\text { t value }\end{array}$ \\
\hline & $(\mathrm{N}=8)$ & $(\mathrm{N}=8)$ & \\
\hline Poids vif $(\mathrm{g})$ & $1423 \pm 279$ & $1536 \pm 164$ & $1,0 \mathrm{NS}$ \\
\hline $\begin{array}{l}\text { Gras abdominal (g) } \\
\text { en p. cent du poids vif } \\
\text { p. cent live weight }\end{array}$ & $37 \quad 11$ & $10 \quad 4$ & $7,3^{* *}$ \\
\hline Gras abdominal ... & $2,5 \pm 0,40$ & $0,6 \pm 0,27$ & $11,1^{* *}$ \\
\hline Cou ........ & $4,2 \quad 0,36$ & $4,3 \quad 0,27$ & $0,4 \mathrm{NS}$ \\
\hline Abats consommables & $4,9 \quad 0,45$ & $4,6 \quad 0,43$ & $1,3 \mathrm{NS}$ \\
\hline $\begin{array}{l}\text { Carcasse éviscérée } \ldots \ldots \ldots \\
\text { en p. cent carcasse éviscérée } \\
\text { p. cent eviscerated weight }\end{array}$ & $57,9 \quad 1,23$ & $59,1 \quad 1,29$ & $1,9 \mathrm{NS}$ \\
\hline Muscles pectoraux ...... & $18,8 \pm 1,07$ & $20,4 \pm 0,85$ & $3,2^{* *}$ \\
\hline Muscles cuis. + pilons & $24,7 \quad 0,91$ & $26,0 \quad 0,68$ & $3,3^{* *}$ \\
\hline $\begin{array}{l}\text { Muscles pectoraux } \\
+ \text { cuis. }+ \text { pilons } \ldots\end{array}$ & $43,5 \quad 1,55$ & $46,4 \quad 0,87$ & $4,6^{* *}$ \\
\hline $\begin{array}{l}\text { en p. cent ens. cuisses }+ \text { pilons } \\
\text { p. cent thighs }+ \text { drumsticks weight }\end{array}$ & & & \\
\hline Peau . & $7,9 \pm 0,70$ & $8,0 \pm 0,70$ & $0,2 \mathrm{NS}$ \\
\hline Os. & $17,3 \quad 1,20$ & $17,6 \quad 1,43$ & $0,4 \mathrm{NS}$ \\
\hline Gras dissécable & $6,0 \quad 0,68$ & $2,9 \quad 0,86$ & $8,0^{* *}$ \\
\hline Muscles & $62,6 \quad 1,65$ & $65,3 \quad 1,14$ & $3,8^{* *}$ \\
\hline Tendons + pertes de dissection & $6,2-$ & $6,2-$ & - \\
\hline
\end{tabular}

Le calcul du pourcentage du poids des muscles pectoraux et des muscles des cuisses et pilons par rapport au poids de la carcasse éviscérée permet de mesurer le rendement en viande «noble» indépendamment du rendement à l'abattage. Ces pourcentages sont plus élevés chez les poulets de la lignée maigre : l'écart se situe entre 2 et 3 p. 100 pour le total des muscles disséqués. L'impact pratique de ce résultat est moins évident que pour le rendement à l'abattage. Il peut cependant intéresser les abattoirs qui font de la découpe ; pour le consommateur qui achète un seul poulet à la fois, il montre qu'à une carcasse moins grasse s'ajoute l'assurance d'avoir un peu plus de viande de premier choix.

La dissection plus complète des cuisses et pilons qui a été faite permet d'avoir une idée de la répartition des différents tissus, sachant que l'ensemble cuisses + pilons est une bonne image de la carcasse entière (RICARD, 1972). Les résultats montrent que les pourcentages d'os et de peau (sans le gras sous-cutané) ne varient pas de façon significative entre les 2 types de poulets, bien que le pourcentage d'os soit un peu plus élevé dans la lignée maigre. L'importance du gras dissécable est plus faible dans la lignée maigre, ce qui se comprend facilement, mais on note que l'écart 
entre les deux lignées est plus faible que pour le gras abdominal. Corrélativement, la part des muscles est plus grande dans la lignée maigre ce qui, là encore, correspond pour une grande part à la diminution du gras dissécable.

L'âge d'abattage de nos animaux correspond au stade où s'effectue la mesure de sélection. Simon \& Leclerce (sous presse) ont résumé une étude longitudinale de l'engraissement faite sur les deux lignées : dès 2 semaines, le dépôt gras abdominal (exprimé en p. 100 du poids vif) est augmenté significativement dans la lignée grasse et la différence est maximale à 9 semaines, âge de la mesure de sélection. Par la suite, ce dépôt augmente parallèlement chez les femelles des 2 lignées tandis que la différence tend à diminuer chez les mâles. Il serait intéressant d'avoir des chiffres comparables pour les différents tissus (en particulier le muscle) de façon à mettre en évidence une éventuelle différence de précocité de ces tissus dans les 2 lignées.

Au stade où nous nous sommes placés, qui correspond au stade de l'abattage commercial des poulets, les résultats des 3 expériences sont cohérents et intéressants : une sélection pour diminuer l'importance relative des dépôts gras abdominaux donne des poulets qui ont une meilleure qualité de carcasse. On peut retenir les chiffres suivants : dépôts gras diminués de moitié par rapport à la lignée grasse, rendement à l'abattage augmenté de 1 à 2 p. 100, rendement en viande augmenté de 2 à 3 p. 100.

Reçu pour publication en juin 1982

\section{Summary}

Meat yield of broilers from two lines of chickens selected on abdominal fat content

A divergent selection experiment on abdominal fat content gave rise to two lines of broilers widely differing in fatness score. Three independant comparisons are reported totaling 52 and 63 birds respectively for the fat and the lean lines. The three sets of data agree with the conclusion that breeding for lowering abdominal fat depot gives a better chicken carcass quality. Fat depots in the lean line are twice less compared to the fat line; slaughter yield (percentage of eviscerated carcass) is increased by 1 to $2 \mathrm{p}$. 100 ; meat yield (percentage of pectoral, thighs and drumsticks muscles in the eviscerated carcass) is increased by 2 to $3 \mathrm{p} .100$.

\section{Références bibliographiques}

DElPECH P., RiCARD F.H., 1965. Relation entre les dépôts adipeux viscéraux et les lipides corporels chez le poulet. Ann. Zootech., 14, 181-189.

LeClerCQ B., Blum J.C., BoYer J.P., 1980. Selecting broilers for low or high abdominal fat : initial observations. Br. Poult. Sci., 21, 107-113.

RiCARD F.N., 1972. Etude de la composition anatomique du poulet. IV. - Ann. Zootech., 21, 49-57.

RiCARD F.H., Rouvier R., 1967. Etude de la composition anatomique du poulet. I. - Ann. Zootech., 16, 23-39.

Simon J., Leclerce B., sous presse. Relation insuline-adiposité dans deux lignées de poulets rendus maigres ou gras par sélection. Repr. Nutr. Develop. 